\title{
Novel derivatives of the antibiotic NHC-Ag(I) drug candidate SBC3: Synthesis, biological evaluation and ${ }^{109} \mathrm{Ag}$ NMR studies
}

\author{
Cillian O’Beirne ${ }^{\mathrm{a}}$, Hessah T. Althani ${ }^{\mathrm{a}}$, Oyinlola Dada ${ }^{\mathrm{a}}$, Jennifer Cassidy ${ }^{\mathrm{a}}$, Kevin Kavanagh ${ }^{\mathrm{b}}$, \\ Helge Müller-Bunz ${ }^{a}$, Yannick Ortin ${ }^{a}$, Xiangming Zhu ${ }^{a}$, Matthias Tacke ${ }^{\text {a,* }}$ \\ ${ }^{a}$ School of Chemistry, University College Dublin, Belfield, Dublin 4, Ireland \\ ${ }^{\mathrm{b}}$ Department of Biology, NUI Maynooth, Ireland
}

\section{A R T I C L E I N F O}

\section{Article history:}

Received 20 February 2018

Accepted 12 April 2018

Available online 22 April 2018

\section{Keywords:}

NHC-silver(I) acetate complexes

MRSA

Antibiotic resistance

${ }^{109} \mathrm{Ag}$ NMR

XRD structures

\begin{abstract}
A B S T R A C T
The synthesis of six novel $N$-heterocyclic carbene silver(I) acetate complexes, three symmetrical and three non-symmetrical, were achieved using 4,5-diphenylimidazole to produce intermediate imidazolium salts and then obtain the corresponding silver(I) complexes through complexation with silver acetate via the Youngs' method. In vitro biological testing, using the Kirby-Bauer disk diffusion method, was conducted against Methicillin-resistant Staphylococcus aureus (MRSA) and Escherichia coli, with a NHC-silver(I) acetate compound, SBC3, and Tetracycline as standards. Silver(I) acetate complex 7 resulted in a $4 \mathrm{~mm}$ clearance against MRSA, showing the highest antibiotic activity of the novel derivatives. Crystallographic data revealed similar bond lengths and angles to previously reported NHC-silver(I) acetate complexes, with complex 8 showing interesting $\eta^{2}$-coordination between the silver atom and acetate oxygens. ${ }^{109} \mathrm{Ag}$ NMR studies were conducted, highlighting the effects of the substituents of the imidazole ring on the silver atom shown by the corresponding shifts in the ${ }^{109} \mathrm{Ag}$ NMR spectra. The incorporation of isopropyl groups to several of the novel complexes resulted in larger upfield ${ }^{109} \mathrm{Ag} \mathrm{NMR}$ shift values compared to all other substituents.
\end{abstract}

(c) 2018 Elsevier Ltd. All rights reserved.

\section{Introduction}

Antimicrobial resistance has become a global phenomenon [1,2] and with cornerstone antibiotic classes becoming less effective $[3,4]$ there is increasing need for new resistance breaking antibiotic compounds. In order to overcome developing resistance, future generation of antibiotics will have to employ multiple mechanisms of action to decrease the chance of such resistance developing, as well as break current resistant strains [5].

For this purpose, silver is a reliable choice for incorporation into a new antibiotic class. With recent research highlighting that its combination with frontline antibiotics can increase their potency [6]. The decades of clinical use as burn wound creams have resulted in infrequent reports of sustained silver resistance, which could be contributed to silver's multiple mechanisms of bactericidal action, including transport protein disruption and redox enzyme inhibition $[7,8]$.

To utilise and enhance silver's proven antibiotic ability, $\mathrm{N}$-heterocyclic carbenes (NHCs) can be incorporated as supplementary

\footnotetext{
* Corresponding author.

E-mail address: matthias.tacke@ucd.ie (M. Tacke).
}

biomolecules. Since the isolation of a stable, singlet carbene by Arduengo [9], $\mathrm{N}$-heterocyclic carbene research has dramatically increased in areas of catalysis and medicinal chemistry [12]. The work of Youngs in the mid-2000's then highlighted the usage of NHC-silver(I) acetate compounds derived from xanthine against bacterial pathogens in the lungs [10]. Since this work, the knowledge base of $\mathrm{NHC}-\mathrm{Ag}(\mathrm{I})$ complexes has been greatly expanded and reviewed extensively [11,12,22].

In order to increase antibacterial ability, the transport of NHCsilver(I) complexes into bacterial cells is an important factor. The large derivability of imidazole-based NHCs [15,24-28] allows for the incorporation of substituent groups that can bestow improved bioavailability and electronically influence the slow release of silver cations in the intracellular environment through the strong $\sigma$-bonding of the NHC [13].

Lipophilic character of the stabilising NHC ligand has been shown to be a key characteristic in NHC-Au(I) anticancer compounds $[14,21]$. Previous research by our group has led to the hypothesis that lipophilic substituents are a large factor by which the antibiotic compound SBC3, a silver(I) complex containing four lipophilic phenyl rings (Fig. 1) [15], is so effective against grampositive bacteria such as Staphylococcus aureus and its widespread 


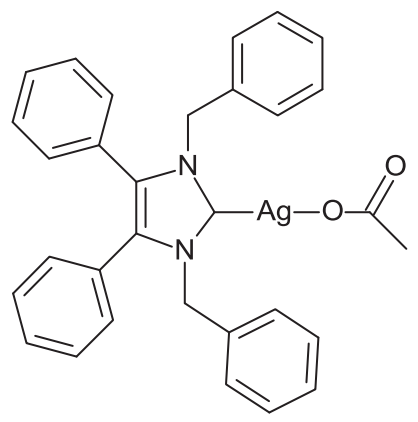

Fig. 1. 1,3-Dibenzyl-4,5-diphenylimidazol-2-ylidene silver(I) acetate (SBC3) [15].

resistant strain, Methicillin-resistant Staphylococcus aureus (MRSA) [16,17].

In an attempt to increase the antibacterial activity of this NHCsilver(I) acetate compound class, the molecular weight was decreased in six new NHC-silver(I) acetate compound. The incorporation of lower molecular weight alkyl chains will improve the drug-like character of the new complexes [18], whilst retaining some lipophilic character that is hypothesized to make the previous SBC3 complex so effective [15]. To assess this hypothesis, all complexes were tested for antibacterial activity using the Kirby-Bauer disk diffusion method against MRSA and Escherichia coli [19].

In addition to the synthesis and testing of these novel NHC-silver(I) complexes, ${ }^{109} \mathrm{Ag}$ NMR studies were conducted. Silver has two isotopes, ${ }^{107} \mathrm{Ag}$ and ${ }^{109} \mathrm{Ag}$, each with a spin of $1 / 2$ and although ${ }^{107} \mathrm{Ag}$ has a slightly higher natural abundance compared to ${ }^{109} \mathrm{Ag}$ ( $51.8 \%$ versus $48.2 \%$, respectively), the NMR studies are usually done using the ${ }^{109} \mathrm{Ag}$ isotope as it has a higher gyromagnetic ratio. Even if the largest problems remain the low sensitivity and long relaxation times of the silver isotopes [23], the use of ${ }^{109} \mathrm{Ag}$ NMR studies could determine interesting analytical information for this class of NHC-silver(I) compounds.

Crystal structures were obtained for all complexes 3-8 and structural information will be reviewed for any influence on the characteristic bonds observed in these types of NHC-silver(I) complexes. The $\eta^{2}$-coordination between the silver(I) moiety and acetate oxygens are two and the possible argentophilic interactions are two notable phenomena that have previously been reported and were surveyed for in this work [15,24-28].

\section{Experimental}

\subsection{General considerations}

Unless otherwise stated in the synthesis procedures, all chemicals and solvents were used as supplied from commercial sources without further purification or drying. The melting points of the corresponding compounds were measured using a Stuart ${ }^{\mathrm{TM}}$ melting point apparatus SMP10, AC input $230 \mathrm{~V}$ and were uncorrected. ${ }^{1} \mathrm{H}$ and ${ }^{13} \mathrm{C}$ NMR spectra were obtained at room temperature on a Varian VnmrS $400 \mathrm{MHz}$ spectrometer and ${ }^{109} \mathrm{Ag}$ NMR spectra were obtained on an Agilent DD2 $500 \mathrm{MHz}$ spectrometer. All NMR data was collected using $\mathrm{CDCl}_{3}$ as deuterated solvent which contains $0.03 \%(\mathrm{v} / \mathrm{v})$ TMS. ${ }^{1} \mathrm{H}$ NMR chemical shifts were referenced to TMS. All other spectra were referenced using absolute referencing from a referenced proton spectrum. For obtaining ${ }^{109} \mathrm{Ag}$ NMR spectra, a ${ }^{1} \mathrm{H}$ NMR measurement was simultaneously obtained using $\mathrm{CDCl}_{3}$ as the deuterated solvent and Tetramethylsilane (TMS) as internal references. Total elemental analysis was undertaken using an Exeter Analytical CE-440 elemental analyser. Infrared spectra were obtained using a Bruker ALPHA Platinum ATR spectrometer.

\subsection{Synthesis}

All synthesis procedures carried out at over $100^{\circ} \mathrm{C}$ were undertaken in sealed, pressurised containers. All silver complex synthesises were carried out under nitrogen and in lightproof flasks. Several silver complexes were purified using Davisil ${ }^{\circledR}$ 40-63 micron silica using pencil columns fashioned from Pasteur pipettes, with dichloromethane used as the eluent.

\subsection{Synthesis of 1-methyl-3-benzyl-4,5-diphenylimidazolium bromide} (1)

1-Methyl-4,5-diphenyl Imidazole $(0.234 \mathrm{~g}, 1.00 \mathrm{mmol})$ and benzyl bromide $(0.12 \mathrm{ml}, 1.00 \mathrm{mmol})$ were placed in $30 \mathrm{ml}$ acetonitrile and stirred under reflux at $85{ }^{\circ} \mathrm{C}$ for $24 \mathrm{~h}$. The solution was filtered and washed with $10 \mathrm{ml}$ acetonitrile. The filtrate was concentrated down to $3 \mathrm{ml}$ and then $50 \mathrm{ml}$ diethyl ether was added. The product was then filtered and dried in vacuo for $2 \mathrm{~h}$ to produce an off-white solid $(0.367 \mathrm{~g}, 90 \%$ yield $)$.

${ }^{1} \mathrm{H}$ NMR (399.899 MHz, $\left.\mathrm{CDCl}_{3}, \mathrm{ppm}\right): 11.00$ (1H, NCHN, s), 7.48$7.36\left(6 \mathrm{H}, \mathrm{CH}_{\text {Arom }}, \mathrm{m}\right), 7.28-7.27\left(3 \mathrm{H}, \mathrm{CH}_{\text {Arom }}, \mathrm{m}\right), 7.22\left(2 \mathrm{H}, \mathrm{CH}_{\text {Arom }}\right.$, dd, 8, $1.5 \mathrm{~Hz}), 7.16-7.12\left(4 \mathrm{H}, \mathrm{CH}_{\text {Arom }}, \mathrm{m}\right), 5.48\left(2 \mathrm{H}, \mathrm{CH}_{2}, \mathrm{~s}\right), 3.96$ $\left(3 \mathrm{H}, \mathrm{CH}_{3}, \mathrm{~s}\right)$.

${ }^{13} \mathrm{C}$ NMR $\left(100.553 \mathrm{MHz}, \mathrm{CDCl}_{3}, \mathrm{ppm}\right): 137.8,133.2,132.5,131.7$, $130.8,130.5,130.4,130.2,129.3,129.2,129.1,128.6,124.8,124.4$ $\left(\mathrm{C}_{\text {Arom }}\right), 51.2\left(\mathrm{CH}_{2}\right), 35.1\left(\mathrm{CH}_{3}\right)$.

Elemental Analysis calculated for $\mathrm{C}_{23} \mathrm{H}_{21} \mathrm{~N}_{2} \mathrm{Br}$ : Calculated: C, 68.15; H, 5.22; N, 6.91; Found: C, 67.80; H, 5.15; N, 6.63.

IR $\left(\mathrm{KBr}, \mathrm{cm}^{-1}\right)$ : $3117(\mathrm{w}), 2998(\mathrm{w}, \mathrm{v}), 1560(\mathrm{w}), 1441(\mathrm{w}), 766$ (m), $736(\mathrm{~m}), 700$ (s), $517(\mathrm{w}), 463(\mathrm{w})$.

Melting point: $239-240{ }^{\circ} \mathrm{C}$.

\subsection{Synthesis of 1-isopropyl-3-benzyl-4,5-diphenylimidazolium bromide (2)}

1-Isopropyl-4,5-diphenyl Imidazole $(0.262 \mathrm{~g}, 1.00 \mathrm{mmol})$ and benzyl bromide $(0.14 \mathrm{ml}, 1.2 \mathrm{mmol})$ were placed in $20 \mathrm{ml}$ acetonitrile and stirred for $3 \mathrm{~d}$. The solution was filtered and washed with $10 \mathrm{ml}$ acetonitrile. The filtrate was concentrated down to $3 \mathrm{ml}$ and $80 \mathrm{ml}$ diethyl ether was added. The resulting precipitate was allowed to settle for $10 \mathrm{~min}$ before being filtered in vacuo for $2 \mathrm{~h}$ to retrieve an off-white solid. $(0.230 \mathrm{~g}, 54 \%$ yield $)$.

${ }^{1} \mathrm{H}$ NMR (399.899 MHz, CDCl 3 , ppm): 11.25 (1H, NCHN, s), 7.46$7.31\left(6 \mathrm{H}, \mathrm{CH}_{\text {Arom }}, \mathrm{m}\right), 7.25-7.20\left(5 \mathrm{H}, \mathrm{CH}_{\text {Arom }}, \mathrm{m}\right), 7.15-7.10(4 \mathrm{H}$, $\left.\mathrm{CH}_{\text {Arom }}, \mathrm{m}\right), 5.67\left(2 \mathrm{H}, \mathrm{CH}_{2}, \mathrm{~s}\right), 4.45\left(1 \mathrm{H}, \mathrm{CH}_{\text {Isopropyl, }}\right.$ hept, $\left.7 \mathrm{~Hz}\right)$, $1.70\left(6 \mathrm{H}, \mathrm{CH}_{3 \text { Isopropyl }}, \mathrm{d}, 1.5 \mathrm{~Hz}\right)$.

${ }^{13} \mathrm{C}$ NMR (100.553 MHz, CDCl $\left.3, \mathrm{ppm}\right): 136.0,133.8,131.8,131.5$, $130.8,130.5,130.4,130.4,129.2,129.1,128.9,128.8,125.0,124.4$ $\left(\mathrm{C}_{\text {Arom }}\right), 51.6\left(\mathrm{CH}_{\text {Isopropyl }}\right), 51.1\left(\mathrm{CH}_{2}\right.$ Benzyl $), 23.5\left(\mathrm{CH}_{3}\right.$ Isopropyl $)$.

Elemental Analysis calculated for $\mathrm{C}_{25} \mathrm{H}_{25} \mathrm{~N}_{2} \mathrm{Br}$ : Calculated: $\mathrm{C}$, 69.28; H, 5.81; N, 6.46; Found: C, 68.35; H, 5.71; N, 6.43.

IR (KBr, cm $\left.{ }^{-1}\right): 3095(\mathrm{w}), 2973(\mathrm{w}, \mathrm{v}), 1547(\mathrm{~m}), 1452(\mathrm{w}), 1183$ (w), $769(\mathrm{~m}), 737$ (s), $706(\mathrm{~m}, \mathrm{v}), 521(\mathrm{w}), 459(\mathrm{w})$.

Melting point: $217-218^{\circ} \mathrm{C}$.

\subsection{General procedure for silver(I) complexes 3-8}

$1.00 \mathrm{mmol}$ of precursor imidazolium salt was stirred with 2.00 mmol of silver acetate $(0.333 \mathrm{~g})$ in $50 \mathrm{ml}$ of dry dichloromethane for $3 \mathrm{~d}$. The reaction mixture was filtered, concentrated to $3 \mathrm{ml}$ and $50 \mathrm{ml}$ diethyl ether was added. The resulting solution was placed in a freezer at $-4{ }^{\circ} \mathrm{C}$ for $12 \mathrm{~h}$ and the resulting solid filtered and dried in vacuo for $4 \mathrm{~h}$ to yield complexes 3-8. 
2.5.1. Synthesis of 1,3-dimethyl-4,5-diphenylimidazol-2-ylidene silver

(I) acetate (3)

Silver(I) complex 3 was prepared as an off white solid. (0.227 g, mmol, 55\% yield).

${ }^{1} \mathrm{H}$ NMR (399.899 MHz, $\left.\mathrm{CDCl}_{3}, \mathrm{ppm}\right)$ : 7.36-7.34 (6H, $\mathrm{CH}_{\text {Arom, }}$ dd, 5, $2 \mathrm{~Hz}$ ), 7.19-7.16 (4H, $\left.\mathrm{CH}_{\text {Arom }}, \mathrm{m}\right), 3.73$ (6H, CH3 $\left.3_{\text {Methyl }}, \mathrm{s}\right), 2.11$ $\left(3 \mathrm{H}, \mathrm{CH}_{3}\right.$ AgOAc, $\left.\mathrm{s}\right)$.

${ }^{13} \mathrm{C}$ NMR $\left(100.553 \mathrm{MHz}, \mathrm{CDCl}_{3}, \mathrm{ppm}\right): 179.2(\mathrm{NCN}), 132.2,130.3$, 129.1, 128.8, 127.9 ( $\left.\mathrm{C}_{\text {Arom }}\right), 37.5$ ( $\left.\mathrm{C}_{\text {Methyl }}\right), 22.9$ ( $\left.\mathrm{C}_{\text {AgOAc }}\right)$.

${ }^{109} \mathrm{Ag}$ NMR (23.278 MHz, $\left.\mathrm{CDCl}_{3}, \mathrm{ppm}\right): 476$.

Elemental Analysis calculated for $\mathrm{C}_{19} \mathrm{H}_{19} \mathrm{~N}_{2} \mathrm{AgO}_{2}$ : Calculated: $\mathrm{C}$, 54.96; H, 4.61; N, 6.75; Found: C, 54.00; H, 4.46; N, 6.55.

IR (KBr, cm $\left.{ }^{-1}\right)$ : $3535(\mathrm{w}, \mathrm{v}), 3053(\mathrm{w}, \mathrm{v}), 2951(\mathrm{w}, \mathrm{v}), 1567$ (s), 1374 (s), 1328 (s), 1012 (w), 765 (s), 698(s), 664 (s), 625 (s), 515 $(\mathrm{m}, \mathrm{v})$

Melting point: $198-201^{\circ} \mathrm{C}$.

\subsubsection{Synthesis of 1,3-diethyl-4,5-diphenylimidazol-2-ylidene silver(I)} acetate (4)

Silver(I) complex 4, was prepared as a light brown solid (0.156 g, 36\% yield).

${ }^{1} \mathrm{H}$ NMR (399.899 $\left.\mathrm{MHz}, \mathrm{CDCl}_{3}, \mathrm{ppm}\right)$ : 7.35-7.33 (6H, $\mathrm{CH}_{\text {Arom, }} \mathrm{dd}$, 5, $2 \mathrm{~Hz}), 7.20-7.18\left(4 \mathrm{H}, \mathrm{CH}_{\text {Arom }}, \mathrm{m}\right), 4.13\left(4 \mathrm{H}, \mathrm{CH}_{2}\right.$ Ethyl, q, $\left.7 \mathrm{~Hz}\right)$, $2.12\left(3 \mathrm{H}, \mathrm{CH}_{3}\right.$ AgOAc, s), $1.23\left(6 \mathrm{H}, \mathrm{CH}_{3}\right.$ Ethyl, t, $\left.7 \mathrm{~Hz}\right)$.

${ }^{13} \mathrm{C}$ NMR (100.553 MHz, CDCl 3 , ppm): $179.2(\mathrm{NCN}), 131.6,130.4$, 129.1, 128.7, 128.2 ( $\left.\mathrm{C}_{\text {Arom }}\right), 44.9\left(\mathrm{CH}_{2}\right.$ Ethyl $), 22.9\left(\mathrm{CH}_{3 \text { AgOAc }}\right), 17.3$ $\left(\mathrm{CH}_{3}\right.$ Ethyl $)$.

${ }^{109} \mathrm{Ag}$ NMR (23.278 MHz, $\mathrm{CDCl}_{3}, \mathrm{ppm}$ ): 480.

Elemental Analysis calculated for $\mathrm{C}_{21} \mathrm{H}_{23} \mathrm{~N}_{2} \mathrm{AgO}_{2}$ : Calculated: $\mathrm{C}$ 56.89; H, 5.22; N, 6.31; Found: C, 57.35; H, 5.31; N, 6.12.

IR (KBr, cm $\left.{ }^{-1}\right): 3052(\mathrm{w}, \mathrm{v}), 2925(\mathrm{w}, \mathrm{v}), 1578(\mathrm{~m}, \mathrm{v}), 1400(\mathrm{~m})$, $1340(\mathrm{~m}, \mathrm{v}), 767$ (w,v), $699(\mathrm{~s}), 666(\mathrm{~m})$.

Melting point: $212-215^{\circ} \mathrm{C}$.

2.5.3. Synthesis of 1,3-diisopropyl-4,5-diphenylimidazol-2-ylidene silver(I) acetate (5)

Silver(I) complex 5, was prepared as a light brown solid. $(0.120$ g, $26 \%$ yield).

${ }^{1} \mathrm{H}$ NMR (399.899 MHz, $\left.\mathrm{CDCl}_{3}, \mathrm{ppm}\right):$ 7.34-7.31 $\left(\mathrm{CH}_{\mathrm{Arom}}, 6 \mathrm{H}, \mathrm{m}\right)$, 7.17-7.14 ( $\left.\mathrm{CH}_{\text {Arom }}, 4 \mathrm{H}, \mathrm{m}\right), 4.40\left(\mathrm{CH}_{\text {Isopropyl, }} 2 \mathrm{H}, 7 \mathrm{~Hz}\right.$, hept $), 2.13$ $\left(\mathrm{CH} 3_{\mathrm{AgOAc}}, 3 \mathrm{H}, \mathrm{s}\right), 1.66\left(\mathrm{CH}_{3}\right.$ Isopropyl, $\left.12 \mathrm{H}, 7 \mathrm{~Hz}, \mathrm{~d}\right)$.

${ }^{13} \mathrm{C}$ NMR (100.553 MHz, $\left.\mathrm{CDCl}_{3}, \mathrm{ppm}\right): 178.8$ (NCN); 130.8, 128.9, 128.6, $128.4\left(\mathrm{C}_{\text {Arom }}\right), 50.2\left(\mathrm{CH}_{\text {Isopropyl }}\right), 24.7\left(\mathrm{CH}_{3}\right.$ Isopropyl $)$, $22.8\left(\mathrm{CH}_{3}\right.$ AgOAC).

${ }^{109} \mathrm{Ag}$ NMR (23.278 MHz, $\mathrm{CDCl}_{3}$, ppm): 533.

Elemental Analysis calculated for $\mathrm{C}_{23} \mathrm{H}_{27} \mathrm{~N}_{2} \mathrm{AgO}_{2}$ : Calculated: $\mathrm{C}$, 58.60; H, 5.77; N, 5.94; Found: C, 58.83; H, 5.85; N, 5.87.

IR (KBr, cm $\left.{ }^{-1}\right): 3055(\mathrm{w}, \mathrm{v}), 2973(\mathrm{w}, \mathrm{v}), 1715(\mathrm{w}), 1368(\mathrm{~m})$, $1329(\mathrm{~m}), 1113(\mathrm{w}), 760(\mathrm{~m}), 699$ (s), $616(\mathrm{~m}), 523(\mathrm{w})$.

Melting point: $178^{\circ} \mathrm{C}$.

2.5.4. Synthesis of 1-isopropyl-3-methyl-4,5-diphenylimidazol-2ylidene silver(I) acetate (6)

Silver(I) complex 6, was prepared as a light brown solid. (0.110 g, $24 \%$ yield).

${ }^{1} \mathrm{H}$ NMR (399.899 MHz, $\left.\mathrm{CDCl}_{3}, \mathrm{ppm}\right):$ 7.37-7.31 (6H, $\left.\mathrm{CH}_{\text {Arom }}, \mathrm{m}\right)$, 7.18-7.15 (4H, $\left.\mathrm{CH}_{\text {Arom }}, \mathrm{m}\right), 4.34\left(1 \mathrm{H}, \mathrm{CH}_{\text {Isopropyl, }}\right.$ Pent, $\left.7 \mathrm{~Hz}\right), 3.78$ $\left(3 \mathrm{H}, \mathrm{CH}_{3}\right.$ Methyl, $\left.\mathrm{s}\right), 2.12\left(3 \mathrm{H}, \mathrm{CH}_{3}\right.$ AgOAc), $1.65\left(6 \mathrm{H}, \mathrm{CH}_{3}\right.$ Isopropyl, d, $7 \mathrm{~Hz})$

${ }^{13} \mathrm{C}$ NMR (100.553 MHz, $\left.\mathrm{CDCl}_{3}, \mathrm{ppm}\right): 179.1$ (NCN), 130.8, 130.2, $129.2,128.9,128.8,128.6,128.0\left(\mathrm{C}_{\text {Arom }}\right), 49.9\left(\mathrm{CH}_{2 \text { Benzyl }}\right), 24.8\left(\mathrm{CH}_{3}\right.$ Isopropyl $), 22.9\left(\mathrm{CH}_{3} \mathrm{AgOAc}\right)$.

${ }^{109} \mathrm{Ag}$ NMR (23.278 MHz, $\left.\mathrm{CDCl}_{3}, \mathrm{ppm}\right): 499$.

Elemental Analysis calculated for $\mathrm{C}_{21} \mathrm{H}_{23} \mathrm{~N}_{2} \mathrm{AgO}_{2}$ : Calculated: $\mathrm{C}$, 56.88; H, 5.23; N, 6.32; Found: C, 56.55; H, 5.19; N, 6.40.
IR $\left(\mathrm{KBr}, \mathrm{cm}^{-1}\right)$ : $3655(\mathrm{w}), 3056(\mathrm{w}, \mathrm{v}), 2975(\mathrm{w}, \mathrm{v}), 1568(\mathrm{~m})$, 1378 (m,v), 1328 (m,v), $765(\mathrm{~m}), 699(\mathrm{~s}), 665(\mathrm{~m}), 526(\mathrm{w})$.

Melting point: $195-200{ }^{\circ} \mathrm{C}$.

2.5.5. Synthesis of 1-methyl-3-benzyl-4,5-diphenylimidazol-2-ylidene silver(I) acetate (7)

Silver(I) complex 7, was prepared as a light brown solid. (0.130 g, $26 \%$ yield).

${ }^{1} \mathrm{H}$ NMR (399.899 MHz, CDCl 3 , ppm): 7.34-7.30 (4H, $\left.\mathrm{CH}_{\text {Arom }}, \mathrm{m}\right)$, 7.27-7.21 (5H, $\left.\mathrm{CH}_{\text {Arom }}, \mathrm{m}\right), 7.18-7.15\left(2 \mathrm{H}, \mathrm{CH}_{\text {Arom }}, \mathrm{m}\right), 7.02-6.97$ $\left(2 \mathrm{H}, \mathrm{CH}_{\text {Arom }}, \mathrm{m}\right), 5.27\left(2 \mathrm{H}, \mathrm{CH}_{2}\right.$ Benzyl, $\left.\mathrm{s}\right), 3.78\left(3 \mathrm{H}, \mathrm{CH}_{3}\right.$ Methyl, $\left.\mathrm{s}\right)$, $2.09\left(3 \mathrm{H}, \mathrm{CH}_{3} \mathrm{AgOAc}, \mathrm{s}\right)$.

${ }^{13} \mathrm{C}$ NMR (100.553 MHz, $\left.\mathrm{CDCl}_{3}, \mathrm{ppm}\right): 178.8(\mathrm{NCN}), 136.2,132.7$, 132.1, 130.77, 130.3, 129.2, 129.1, 128.7, 128.6, 127.9, 127.9, 127.7, $127.5\left(\mathrm{C}_{\text {Arom }}\right), 53.4\left(\mathrm{C}_{\text {Benzyl }}\right), 37.8$ ( $\left.\mathrm{C}_{\text {Methyl }}\right), 22.5$ ( $\left.\mathrm{C}_{\text {AgOac }}\right)$.

${ }^{109} \mathrm{Ag}$ NMR $\left(23.278 \mathrm{MHz}, \mathrm{CDCl}_{3}, \mathrm{ppm}\right): 476$.

Elemental Analysis calculated for $\mathrm{C}_{27} \mathrm{H}_{27} \mathrm{~N}_{2} \mathrm{AgO}_{2}$ : Calculated: $\mathrm{C}$, 61.77; H, 4.71; N, 5.70; Found: C, 60.82; H, 4.71; N, 5.50.

IR $\left(\mathrm{KBr}, \mathrm{cm}^{-1}\right)$ : $3054(\mathrm{w}, \mathrm{v}), 2920(\mathrm{w}, \mathrm{v}) 1704(\mathrm{w}), 1573$ (m,v), $1379(\mathrm{~m}), 1257$ (m), 735 (s,v), 696 (s), 617 (m), $514(\mathrm{w})$.

Melting point: $118-120^{\circ} \mathrm{C}$.

2.5.6. Synthesis of 1-isopropyl-3-benzyl-4,5-diphenylimidazol-2ylidene silver(I) acetate $(\mathbf{8})$

Silver(I) complex 8, was prepared as a light brown solid. (0.060 g, $12 \%$ yield).

${ }^{1} \mathrm{H}$ NMR (399.899 MHz, $\left.\mathrm{CDCl}_{3}, \mathrm{ppm}\right): 7.35-7.29\left(4 \mathrm{H}, \mathrm{CH}_{\text {Arom }}, \mathrm{m}\right)$, 7.24-7.20 (5H, $\left.\mathrm{CH}_{\text {Arom }}, \mathrm{m}\right), 7.17-7.15\left(2 \mathrm{H}, \mathrm{CH}_{\text {Arom }}, \mathrm{m}\right), 7.00-6.94$ $\left(4 \mathrm{H}, \mathrm{CH}_{\text {Arom }}\right.$ ddd, 12, 7.5, $\left.2.5 \mathrm{~Hz}\right), 5.33\left(2 \mathrm{H}, \mathrm{CH}_{\text {Benzyl }}, \mathrm{s}\right), 4.39(1 \mathrm{H}$, $\mathrm{CH}_{\text {Isopropyl }}$, hept, $\left.7 \mathrm{~Hz}\right), 2.10\left(3 \mathrm{H}, \mathrm{CH}_{3} \mathrm{AgOAc}, \mathrm{s}\right), 1.70\left(6 \mathrm{H}, \mathrm{CH}_{3}\right.$ Isopropyl, d, $7 \mathrm{~Hz}$ ).

${ }^{13} \mathrm{C}$ NMR (100.553 MHz, $\left.\mathrm{CDCl}_{3}, \mathrm{ppm}\right): 179.0(\mathrm{NCN}), 136.3,130.8$, 130.7, 129.2, 128.9, 128.7, 128.6, 128.5, 128.0, 127.9, 127.9, 127.3 $\left(\mathrm{C}_{\text {Arom }}\right), 54.5\left(\mathrm{C}_{\text {Benzyl }}\right), 50.1\left(\mathrm{CH}_{\text {isopropyl }}\right), 24.8\left(\mathrm{CH}_{3}\right.$ isopropyl $), 22.8$ $\left(\mathrm{C}_{\mathrm{AgOAC}}\right)$.

${ }^{109} \mathrm{Ag}$ NMR (23.278 MHz, $\left.\mathrm{CDCl}_{3}, \mathrm{ppm}\right): 506$.

Elemental Analysis calculated for $\mathrm{C}_{27} \mathrm{H}_{27} \mathrm{~N}_{2} \mathrm{AgO}_{2}$ : Calculated: $\mathrm{C}$, 62.43; H, 5.23; N, 5.39; Found: C, 62.05; H, 5.08; N, 5.16.

IR $\left(\mathrm{KBr}, \mathrm{cm}^{-1}\right)$ : $3056(\mathrm{w}, \mathrm{v}), 2972(\mathrm{w}, \mathrm{v}), 1716(\mathrm{w}), 1570(\mathrm{~m})$, $1375(\mathrm{~m}), 1329(\mathrm{~m}), 700(\mathrm{~s}), 666(\mathrm{~s}), 617(\mathrm{~m}), 576(\mathrm{~m})$.

Melting point: $176-178^{\circ} \mathrm{C}$.

\subsection{Antibacterial studies}

Preliminary in vitro biological studies were carried out to assess the antibacterial effect of the newly synthesised symmetrical and non-symmetrical derivatives of SBC3 against two pathogenic bacterial strains. Methicillin-Resistant Staphylococcus aureus (ATCC 43300) was chosen as the Gram-positive bacterial test strain and Escherichia coli (ATCC 25922) chosen as the Gram-negative bacterial test strain.

To test the biological activity of the novel complexes, 3-8, the Kirby-Bauer disk diffusion method was used [19]. Both bacterial strains were cultured in $2 \mathrm{ml}$ LB medium from single colonies at $37^{\circ} \mathrm{C}$ for $24 \mathrm{~h}$ whilst shaking. All antibacterial work was carried out under sterile conditions.

For both bacterial strains, $50 \mu \mathrm{L}$ of culture was spread over agarLB medium in a petri plate. To each plate, $5.5 \mathrm{~mm}$ Whatman paper disks were evenly placed into four marked quadrants. Stock solutions of $1 \mathrm{mg} / \mathrm{ml}$ of each silver complex in DMSO were made, with two volumes of $5 \mu \mathrm{L}$ and $10 \mu \mathrm{L}$ tested for each complex against both bacterial strains.

DMSO, without the addition of a silver complex, was used with each round of testing as a control in $5 \mu \mathrm{L}$ and $10 \mu \mathrm{L}$ volumes. Plates were covered and incubated at $37^{\circ} \mathrm{C}$ for $24 \mathrm{~h}$. The area of clearance is defined as the distance between the beginning of bacterial 
growth and the edge of the Whatman disk; the area of inhibition was measured in millimetres ( $\mathrm{mm})$.

\section{Results and discussion}

\subsection{Synthesis}

With the objective of understanding the influence of changing non-polar substituents at the 1 and 3 position of the SBC3 silver (I) complex (Fig. 1), a series of NHC imidazolium salts were synthesized and their corresponding silver(I) complexes were produced. With the incorporation of the silver acetate moiety as the primary antimicrobial agent, the derivatisation of the NHC imidazole backbone was a logical step to try and increase the biological efficacy.

By incorporating alkyl chains at the 1 and 3 positions of the imidazole ring, lower molecular weight silver(I) complexes were synthesized to increase the 'drug-like' character of this class. Six novel lower weight derivatives of the SBC3 molecule have been synthesized (Scheme 1) and tested for antibiotic activity. All silver complexes were characterised by spectroscopic and analytical methods, with elemental analysis of $\mathrm{C}, \mathrm{H}, \mathrm{N}$, and crystal structure data obtained for certainty of structure and purity.

Using 4,5-diphenyl imidazole as the starting material, the mono-alkylated imidazoles of 1-methyl and 1-isopropyl-4,5diphenyl imidazole were synthesized by adjusted literature methods [20]; both were confirmed by NMR spectroscopy and elemental analysis.

The symmetrical imidazolium salt precursors of complexes 3, 4 \& $\mathbf{5}$ were synthesized under high pressure and high temperature conditions with 4,5-diphenyl imidazole and potassium carbonate added to a Teflon container with a small amount of acetonitrile. The corresponding ligand (iodomethane; bromoethane; 2-bromopropane) was then injected before the container was sealed in a stainless steel casing and heated at $120^{\circ} \mathrm{C}$ for 3 days. All reactions were filtered, concentrated and precipitated from solution using diethyl ether in yields of $81 \%, 89 \%$ and $85 \%$ for the precursors of sil$\operatorname{ver}(\mathrm{I})$ complexes 3, 4 and 5, respectively. All imidazolium salts were confirmed through NMR spectroscopy and elemental analysis and compared to literature values [20,29,30].

The non-symmetrical imidazolium salt precursors of complexes 6, 7 \& 8 were synthesized from the previously mentioned monoalkylated imidazoles. The precursor imidazolium salt to complex 6 was synthesized using the same high pressure and high temperature conditions and the same workup procedure as for the salt precursors of 3, 4 \& 5; the imidazolium salt was confirmed by NMR spectroscopy, elemental analysis and compared to literature values [20]. The precursor imidazolium salt for silver(I) complex
7 was synthesized by refluxing 1-methyl-4,5-diphenyl imidazole and benzyl bromide for $24 \mathrm{~h}$, and worked up as the other precursor salts to obtain the desired product in $90 \%$ yield. The precursor imidazolium salt for silver(I) complex 8 was synthesized by stirring 1isopropyl-4,5-diphenyl imidazole and benzyl bromide for 3 days at room temperature. The reaction was worked up as the other precursor salts to obtain the desired imidazolium salt in 54\% yield.

The corresponding silver(I) acetate complexes, 3-8, were all synthesized by stirring the respective precursor imidazolium salt with two equivalents of silver acetate in dry dichloromethane, under nitrogen, in a lightproof flask for 3 days. All products were filtered, washed with dry dichloromethane, concentrated and precipitated with diethyl ether. All complexes were placed in a $-4{ }^{\circ} \mathrm{C}$ freezer for $12 \mathrm{~h}$ to allow greater precipitation to achieve yields between $12 \%$ and $54 \%$. Several of the imidazolium salts proved less reactive than others with silver acetate under these conditions, and the corresponding silver(I) complexes $(\mathbf{4}, \mathbf{6}, \mathbf{7}, \mathbf{8})$ required post-precipitation purification using a pencil column, made from a cotton wool bud and one inch of Davisil ${ }^{\circledR} 40-63$ micron silica inside a Pasteur pipette.

The precursor imidazolium salt to the SBC3 molecule was synthesized in an improved yield of $98 \%$ using a modified literature method [15]. 4,5-Diphenyl imidazole and 2.2 equivalents of benzyl bromide were reacted under reflux at $85{ }^{\circ} \mathrm{C}$ for $24 \mathrm{~h}$ in $50 \mathrm{ml}$ acetonitrile and worked up as previous precursor salts. The SBC3 molecule was synthesized by literature method in a yield of $59 \%$ by reacting silver acetate with the precursor imidazolium salt in dry dichloromethane for 3 days at room temperature, in darkness. The complex was worked up as the other silver(I) complexes.

\subsection{NMR spectroscopy}

All of the imidazolium salt intermediates and silver(I) complexes were fully characterised by ${ }^{1} \mathrm{H},{ }^{13} \mathrm{C}$ NMR spectroscopy, along with further analysis by ${ }^{109} \mathrm{Ag}$ NMR spectroscopy for the silver(I) complexes. All previously published imidazoles and imidazolium

Table 1

Recorded ${ }^{109} \mathrm{Ag}$ NMR resonance shifts for novel silver(I) complexes.

\begin{tabular}{ll}
\hline Complex & ${ }^{109} \mathrm{Ag}$ shift $(\delta \mathrm{ppm})$ \\
\hline SBC3 & 488 \\
$\mathbf{3}$ & 476 \\
$\mathbf{4}$ & 480 \\
$\mathbf{5}$ & 533 \\
$\mathbf{6}$ & 499 \\
$\mathbf{7}$ & 476 \\
$\mathbf{8}$ & 506 \\
\hline
\end{tabular}

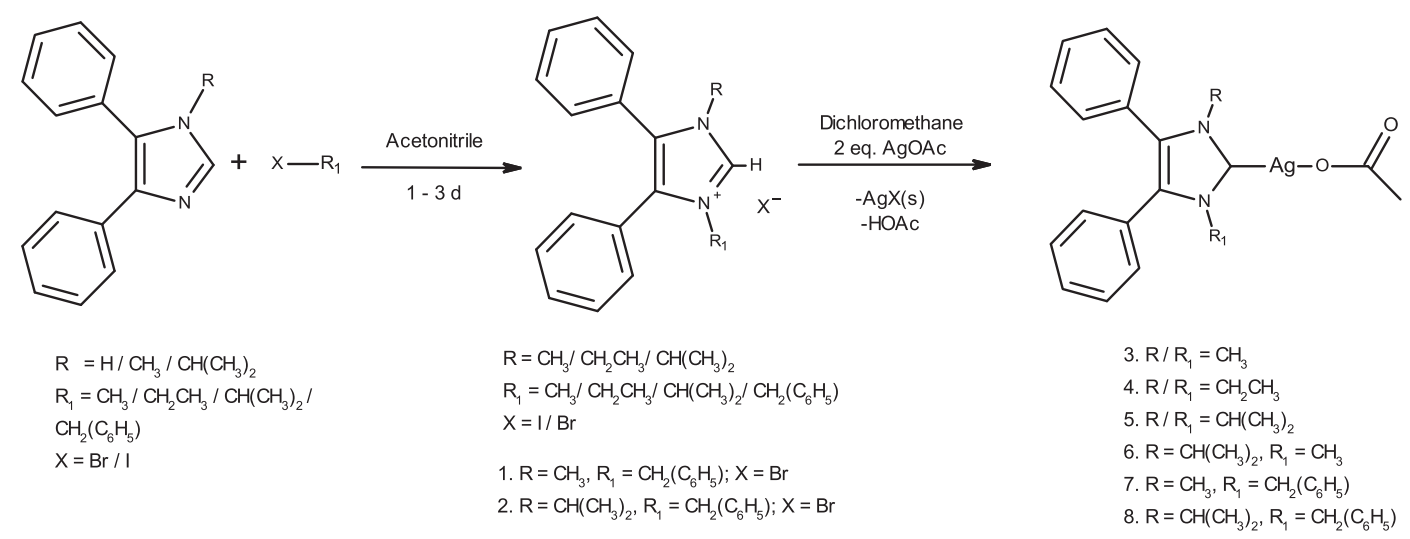

Scheme 1. Synthesis of symmetrical and non-symmetrical imidazolium salt precursors, and their corresponding NHC-silver(I) complexes 3-8 
Table 2

Crystal data and structural refinement information for complexes 3-8.

\begin{tabular}{|c|c|c|c|c|c|c|}
\hline Identification code & 3 & 4 & 5 & 6 & 7 & 8 \\
\hline $\begin{array}{l}\text { Empirical formula } \\
\text { Molecular formula }\end{array}$ & $\begin{array}{l}\mathrm{C}_{19} \mathrm{H}_{23} \mathrm{~N}_{2} \mathrm{O}_{4} \mathrm{Ag} \\
\mathrm{C}_{19} \mathrm{H}_{19} \mathrm{~N}_{2} \mathrm{O}_{2} \mathrm{Ag} \times 2\left(\mathrm{H}_{2} \mathrm{O}\right)\end{array}$ & $\mathrm{C}_{21} \mathrm{H}_{23} \mathrm{~N}_{2} \mathrm{O}_{2} \mathrm{Ag}$ & $\begin{array}{l}\mathrm{C}_{24} \mathrm{H}_{29} \mathrm{~N}_{2} \mathrm{O}_{2} \mathrm{Cl}_{2} \mathrm{Ag} \\
\mathrm{C}_{23} \mathrm{H}_{27} \mathrm{~N}_{2} \mathrm{O}_{2} \mathrm{Ag} \times \mathrm{CH}_{2} \mathrm{Cl}_{2}\end{array}$ & $\begin{array}{l}\mathrm{C}_{43} \mathrm{H}_{48} \mathrm{~N}_{4} \mathrm{O}_{4} \mathrm{Cl}_{2} \mathrm{Ag}_{2} \\
\left(\mathrm{C}_{21} \mathrm{H}_{23} \mathrm{~N}_{2} \mathrm{O}_{2} \mathrm{Ag}\right)_{2} \times \mathrm{CH}_{2} \mathrm{Cl}_{2}\end{array}$ & $\begin{array}{l}\mathrm{C}_{25.64} \mathrm{H}_{24.28} \mathrm{~N}_{2} \mathrm{O}_{2} \mathrm{Cl}_{1.28} \mathrm{Ag} \\
\mathrm{C}_{25} \mathrm{H}_{23} \mathrm{~N}_{2} \mathrm{O}_{2} \mathrm{Ag} \times 0.64 \\
\left(\mathrm{CH}_{2} \mathrm{Cl}_{2}\right)\end{array}$ & $\mathrm{C}_{27} \mathrm{H}_{27} \mathrm{~N}_{2} \mathrm{O}_{2} \mathrm{Ag}$ \\
\hline Formula weight & 451.26 & 443.28 & 556.26 & 971.49 & 545.78 & 519.37 \\
\hline Crystal system & triclinic & triclinic & orthorhombic & triclinic & orthorhombic & triclinic \\
\hline \multirow{4}{*}{$\begin{array}{l}\text { Space group } \\
\text { Unit cell dimensions/Å( }\left(^{\circ}\right)\end{array}$} & $P-1(\# 2)$ & $P-1(\# 2)$ & $P 2_{1} 2_{1} 2_{1}$ (\#19) & $P-1(\# 2)$ & $\operatorname{Pna2}_{1}(\# 33)$ & $P-1(\# 2)$ \\
\hline & $a=8.5705(2) b=9.1007(2)$ & $\begin{array}{l}a=14.60488(6) b=16.07477 \\
(7)\end{array}$ & $\begin{array}{l}a=8.89667(7) b=9.20695 \\
(7)\end{array}$ & $\begin{array}{l}a=8.9450(2) b=9.5119 \\
(2)\end{array}$ & $\begin{array}{l}a=15.1362(2) b=11.37991 \\
(9)\end{array}$ & $a=10.09486(8) b=10.9479(1)$ \\
\hline & $\begin{array}{l}c=13.2387(3) \AA \alpha=99.037 \\
(2)^{\circ}\end{array}$ & $\begin{array}{l}c=18.86008(9) \alpha=70.2548 \\
(4)\end{array}$ & $c=29.3893(3) \alpha=90^{\circ}$ & $\begin{array}{l}c=13.5607(2) \alpha=71.653 \\
(2)^{\circ}\end{array}$ & $c=28.5638(2) \alpha=90^{\circ}$ & $c=12.1433(1) \alpha=96.2856(7)^{\circ}$ \\
\hline & $\begin{array}{l}\beta=105.107(2)^{\circ} \gamma=93.185 \\
(2)^{\circ}\end{array}$ & $\begin{array}{l}\beta=70.2548(4) \gamma=75.3434 \\
\text { (3) }\end{array}$ & $\beta=90^{\circ} \gamma=90^{\circ}$ & $\begin{array}{l}\beta=83.779(2)^{\circ} \gamma=73.152 \\
(2)^{\circ}\end{array}$ & $\beta=90^{\circ} \gamma=90^{\circ}$ & $\begin{array}{l}\beta=111.1459(7)^{\circ} \gamma=105.4602 \\
(8)^{\circ}\end{array}$ \\
\hline$V\left(\AA^{3}\right)$ & $979.47(4)$ & $3915.50(3)$ & 2407.31(4) & $1047.96(4)$ & $4920.07(8)$ & $1174.395(19)$ \\
\hline$Z$ & 2 & 8 & 4 & 1 & 8 & 2 \\
\hline$D_{\text {calc }}\left(\mathrm{Mg} / \mathrm{m}^{3}\right)$ & 1.530 & 1.504 & 1.535 & 1.539 & 1.474 & 1.469 \\
\hline Absorption coefficient $\left(\mathrm{mm}^{-1}\right)$ & 8.471 & 1.046 & 8.941 & 9.035 & 8.045 & 7.084 \\
\hline$F(000)$ & 460 & 1808 & 1136 & 494 & 2215.4 & 532 \\
\hline Crystal size $\left(\mathrm{mm}^{3}\right)$ & $0.241 \times 0.174 \times 0.039$ & $0.325 \times 0.240 \times 0.169$ & $0.2152 \times 0.1344 \times 0.1165$ & $0.202 \times 0.154 \times 0.093$ & $0.197 \times 0.138 \times 0.125$ & $0.194 \times 0.122 \times 0.086$ \\
\hline Theta range for data collection $\left(^{\circ}\right)$ & $3.514-76.848$ & $2.85-33.02$ & $3.01-76.72$ & $3.434-76.849$ & $4.182-77.060^{\circ}$ & $4.010-76.989$ \\
\hline Index ranges & $-10 \leq h \leq 10$ & $-21 \leq h \leq 21$ & $-7 \leq h \leq 11$ & $-11 \leq h \leq 11$ & $-16 \leq h \leq 18$ & $-12 \leq h \leq 12$ \\
\hline & $\begin{array}{l}-11 \leq k \leq 11 \\
-16 \leq l \leq 16\end{array}$ & $\begin{array}{l}-24 \leq k \leq 24 \\
-28 \leq l \leq 28\end{array}$ & $\begin{array}{l}-11 \leq k \leq 11 \\
-36 \leq l \leq 33\end{array}$ & $\begin{array}{l}-11 \leq k \leq 11 \\
-17 \leq l \leq 17\end{array}$ & $\begin{array}{l}-14 \leq k \leq 14 \\
-35 \leq l \leq 35\end{array}$ & $\begin{array}{l}-11 \leq k \leq 13 \\
-15 \leq l \leq 15\end{array}$ \\
\hline Reflections collected & 21048 & 241717 & 17276 & 20542 & $\begin{array}{l}-35 \leq l \leq 35 \\
115490\end{array}$ & $\begin{array}{l}-15 \leq l \leq 15 \\
46914\end{array}$ \\
\hline Independent reflections & $4081\left[R_{\mathrm{int}}=0.0283\right]$ & $28036\left[R_{\mathrm{int}}=0.0397\right]$ & $5001\left[R_{\mathrm{int}}=0.0354\right]$ & $4351\left[R_{\text {int }}=0.0665\right]$ & $10325\left[R_{\text {int }}=0.0454\right]$ & $4920\left[R_{\text {int }}=0.0288\right]$ \\
\hline Completeness to $\theta=67.684^{\circ}$ & $100.00 \%$ & $99.40 \%$ & $99.20 \%$ & $99.80 \%$ & $99.80 \%$ & $100.00 \%$ \\
\hline Absorption correction & gaussian & gaussian & analytical & gaussian & gaussian & gaussian \\
\hline $\begin{array}{l}\text { Maximum and minimum } \\
\text { transmission }\end{array}$ & 0.736 and 0.227 & 0.877 and 0.774 & 0.503 and 0.306 & 0.643 and 0.375 & 0.496 and 0.291 & 0.666 and 0.422 \\
\hline Data/restraints/parameters & $4081 / 0 / 245$ & 28036/0/949 & $5001 / 0 / 285$ & $4351 / 0 / 294$ & $10325 / 1 / 601$ & $4920 / 0 / 330$ \\
\hline Goodness-of-fit (GOF) on $F^{2}$ & 1.041 & 1.088 & 1.019 & 1.117 & 1.059 & 1.084 \\
\hline Final $R$ indices $[I>2 \sigma(I)]$ & $R_{1}=0.0206, w R_{2}=0.0527$ & $R_{1}=0.0306, w R_{2}=0.0714$ & $R_{1}=0.0217, w R_{2}=0.0540$ & $R_{1}=0.0481, w R_{2}=0.1194$ & $R_{1}=0.0482, w R_{2}=0.1413$ & $R_{1}=0.0350, w R_{2}=0.0814$ \\
\hline$R$ indices (all data) & $R_{1}=0.0224, w R_{2}=0.0537$ & $R_{1}=0.0420, w R_{2}=0.0789$ & $R_{1}=0.0228, w R_{2}=0.0546$ & $R_{1}=0.0568, w R_{2}=0.1470$ & $R_{1}=0.0496, w R_{2}=0.1434$ & $R_{1}=0.0398, w R_{2}=0.0857$ \\
\hline $\begin{array}{l}\text { Largest difference peak and hole (e } \\
\AA^{-3} \text { ) }\end{array}$ & 0.442 and -0.507 & 3.186 and -1.328 & 0.650 and -0.502 & 1.924 and -1.869 & 1.404 and -0.855 & 0.905 and -1.589 \\
\hline
\end{tabular}


3



4



5

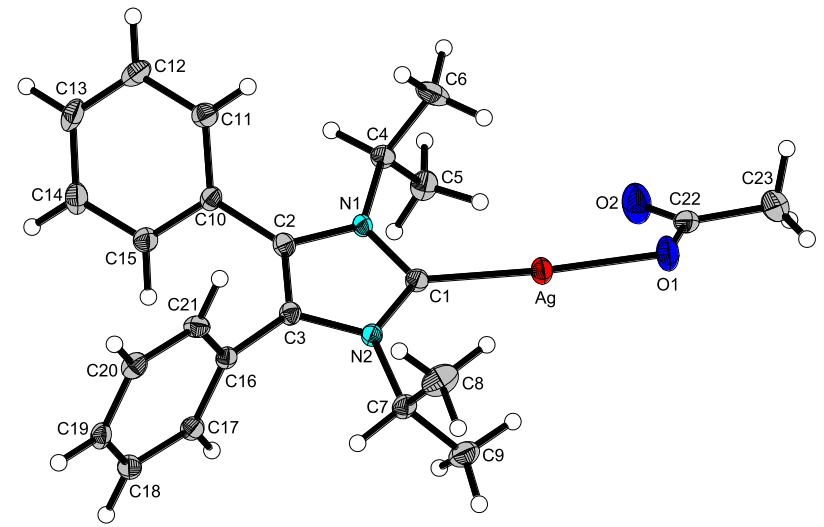

6

$$
7
$$
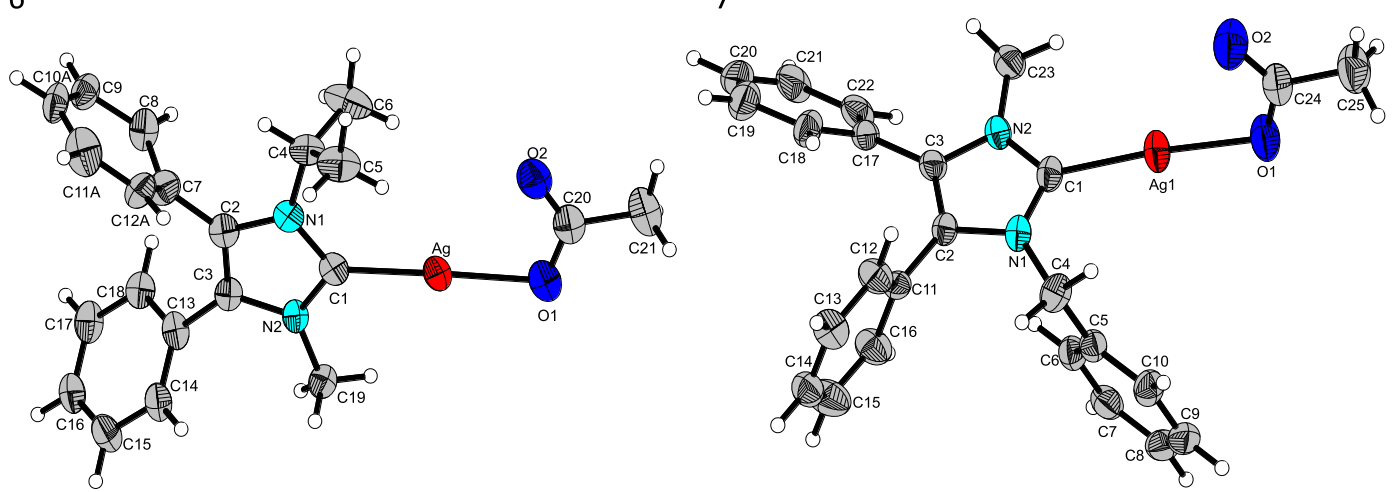

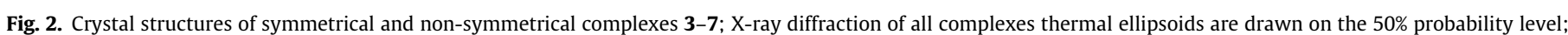
disorder neglected for structure of complex $\mathbf{6}$.

salts were confirmed by comparison with literature values [HYPERLINK "SPS:refid::bib15_bib20_bib27_bib28"15,20,27,28].

In the case of the imidazolium salts, a singlet peak can be clearly seen on the ${ }^{1} \mathrm{H}$ spectra in the range of $\delta=10.38-11.39 \mathrm{ppm}$. This corresponds to literature values for imidazolium salts with halogen anions $\left(\mathrm{Br}^{-}, \mathrm{I}^{-}\right)[15,19,24-28]$, as the proton attached to the carbene carbon of the imidazole ring becomes deshielded due to the positive nature of the imidazolium and presence of the anionic halide counter ion [10].

For all silver(I) complexes, there is the distinctive loss of the carbene proton signal in the range $\delta=10.38-11.39 \mathrm{ppm}$, and there is the presence of the new silver acetate $\mathrm{CH}_{3}$ singlet peak in the range $\delta=2.09-2.13 \mathrm{ppm}$.

For complexes $\mathbf{3 - 8}$, the ${ }^{13} \mathrm{C}$ resonances for the carbene carbon fall within the region of $\delta=177.0-179.2 \mathrm{ppm}$, as seen in previous publication [15]. Along with the shifted carbene signals, the silver acetate $\mathrm{CH}_{3}$ group is the other characteristic peak that is present in the ${ }^{13} \mathrm{C}$ NMR spectrum after complexation, falling in the range of $\delta$ $=22.5-22.9 \mathrm{ppm}$.

${ }^{109} \mathrm{Ag}$ NMR was conducted on all silver complexes and the corresponding broad resonance peaks ( $\delta \mathrm{ppm})$ were found, as shown in Table 1 . The NMR resonances of the silver complexes were determined on a w/v concentration basis, with $100 \mathrm{mg}$ of silver(I) complex to $0.6 \mathrm{ml}$ deuterated chloroform as the solvent.

The different substituent patterns at the 1 and 3 positions of the imidazole ring will directly affect the electronic properties of the carbene carbon. These electronic effects result in differentiations in NMR shifts, as is the case for the carbene hydrogen in the imidazolium salt precursors and now in the shifts of the silver moiety in the complexes.

These values were found within a similar region to a previously reported imidazole $\mathrm{NHC}$-silver(I) chloride complex that resolves a ${ }^{109} \mathrm{Ag}$ NMR signal at $\delta=597 \mathrm{ppm}$, in deuterated acetonitrile [31], and a previously reported dinuclear silver OTf complex resolving 


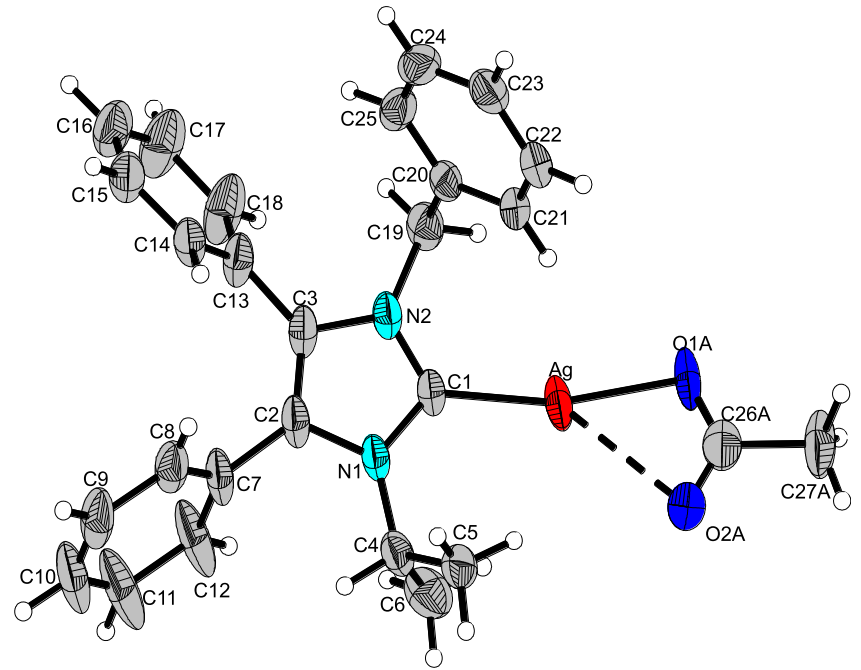

Fig. 3. Crystal structure of complex 8; thermal ellipsoids are drawn on the $50 \%$ probability level, disorder neglected.

a Ag NMR signal at $\delta=541 \mathrm{ppm}$, in deuterated chloroform [32 $\left.{ }^{109}\right]$. No literature values could be found for NHC-silver(I) acetate complexes for direct comparison.

Each ${ }^{109} \mathrm{Ag}$ signal was resolved as a broad signal, despite the relative low sensitivity experiment. The signal for the SBC3 molecule resolved at $\delta=488 \mathrm{ppm}$; with two benzyl groups on the 1 and 3 positions, this was used as the standard comparison against the six novel complexes.

The signals of the three symmetrical silver(I) complexes steadily shift further upfield with the increase in atoms on the carbon chain, with a larger up-field shift when isopropyl groups are incorporated in complex 5. For the non-symmetrical compounds we again see the influence of the isopropyl group compared to the methyl substituent. Comparing the shift of complex 3 ( $\delta=476$ $\mathrm{ppm}$ ) to that of complex 7 ( $\delta=476 \mathrm{ppm}$ ), there does not seem to be a large influence from the benzyl substituent.

The largest downfield shifts seen in this series are associated with the complexes containing isopropyl groups. The jump of 12 $\mathrm{ppm}$ in the silver chemical shift for complex 8 compared to SBC3 seems to result from a single substitution of the benzyl group by an isopropyl one. This jump is even more pronounced with a 55 ppm shift for the complex $\mathbf{5}$ after the outright replacement of benzyl groups with isopropyl substituents. This isopropyl effect seems to indicate a large downfield field shift even with a single replacement of the methyl, ethyl or benzyl substituents with an isopropyl group.

\subsection{IR spectroscopy}

In the IR spectra of all silver complexes there are consistent absorptions in the region of $1560-1580 \mathrm{~cm}^{-1}$ corresponding to the $\mathrm{C}=\mathrm{O}$ stretching vibration of the $\eta^{1}$-bonded acetate group, which is consistent with previously reported complexes [15,19,24-28]. All complexes also show low-intensity absorptions just above and under the $3000 \mathrm{~cm}^{-1}$ region, which are corresponding to aromatic and aliphatic $\mathrm{C}-\mathrm{H}$ stretching vibrations, and strong absorptions in the $695-750 \mathrm{~cm}^{-1}$ region that correspond to the phenyl $\mathrm{C}-\mathrm{H}$ bending vibrations.

\subsection{Crystal structure data}

Crystal data of 3-8 were collected using an Rigaku Oxford Diffraction (former Agilent Technologies, former Oxford Diffraction) SuperNova A diffractometer fitted with an Atlas detector. 4 was measured with Mo $\mathrm{K} \alpha(0.71073 \AA)$, all others with $\mathrm{Cu} \mathrm{K} \alpha$ (1.54184 $\AA$ ). A complete dataset was collected, assuming that the Friedel pairs are not equivalent. An analytical absorption correction based on the shape of the crystal was performed [33]. The structures were solved by direct methods using [34] and refined by full matrix least-squares on F2 for all data using [34sheLXSSHELXL]. Refinement data can be seen in Table 2 .

The hydrogen atoms of the water molecules in $\mathbf{3}$ were located in the difference Fourier map. In subsequent refinements the water molecules were restrained to have the ideal shape using DFIX. After convergence the water molecules were refined as rigid groups. All other hydrogen atoms were added at calculated positions and refined using a riding model. Their isotropic temperature factors were fixed to 1.2 times (1.5 times for methyl groups and water hydrogens) the equivalent isotropic displacement parameters of the parent atom. Anisotropic thermal displacement parameters were used for all non-hydrogen atoms.

Appropriate crystals of complexes 3-8 were obtained for X-ray crystallography by the slow diffusion of pentane into a small volume of a saturated solution of dichloromethane at $2{ }^{\circ} \mathrm{C}$, Figs. 2 and 3.

Selected bond lengths and angles are listed in Table 3. Both the shapes of the imidazole rings and the $\mathrm{Ag}-\mathrm{C}$ bond length agree very well with previously reported values [15,19,24-28].

In all complexes reported here the $\mathrm{Ag}-\mathrm{O}$ bond length is even shorter than the one in the SBC3 molecule [15], with the possible exception of the minor disorder part in 8 . All $\mathrm{C}-\mathrm{Ag}-\mathrm{O}$ bond angles are close to $180^{\circ}$, again with the possible exception of $\mathbf{8}$. There the acetate group is disordered in such a way that both monodentate and bidentate binding to the silver atom seem to be present.

However, the displacement ellipsoid of the silver atom indicates that it may also be disordered. An attempt to refine this led to

Table 3

Selected bond lengths and angles for silver(I) complexes 3-8.

\begin{tabular}{|c|c|c|c|c|c|c|}
\hline & 3 & $4^{a}$ & $\mathbf{5}$ & 6 & $7^{a}$ & 8 \\
\hline \multicolumn{7}{|l|}{ Bond length $(\AA)$} \\
\hline$C(1)-N(1)$ & $1.352(2)$ & $1.352(2)$ & $1.360(3)$ & $1.378(7)$ & $1.346(9)$ & $1.349(3)$ \\
\hline $\mathrm{C}(1)-\mathrm{N}(2)$ & $1.349(3)$ & $1.353(2)$ & $1.355(3)$ & $1.361(6)$ & $1.333(8)$ & $1.344(4)$ \\
\hline$N(1)-C(2)$ & $1.393(2)$ & $1.3928(19)$ & $1.398(3)$ & $1.391(6)$ & $1.406(8)$ & $1.400(4)$ \\
\hline$C(2)-C(3)$ & $1.362(3)$ & $1.365(2)$ & $1.353(3)$ & $1.365(7)$ & $1.358(9)$ & $1.359(5)$ \\
\hline $\mathrm{C}(3)-\mathrm{N}(2)$ & $1.393(2)$ & $1.395(2)$ & $1.398(3)$ & $1.388(6)$ & $1.393(8)$ & $1.397(3)$ \\
\hline $\mathrm{Ag}-\mathrm{C}(1)$ & $2.0705(19)$ & $2.0667(16)$ & $2.080(2)$ & $2.065(5)$ & $2.080(7)$ & $2.073(3)$ \\
\hline $\mathrm{Ag}-\mathrm{O}(1)$ & $2.0986(14)$ & $2.1336(13)$ & $2.1119(18)$ & $2.138(4)$ & $2.109(6)$ & $2.133(4)^{b}$ \\
\hline \multicolumn{7}{|l|}{ Bond angle $\left({ }^{\circ}\right)$} \\
\hline$C(1)-A g-O(1)$ & $172.74(6)$ & $173.36(6)$ & $176.50(8)$ & $167.23(16)$ & $170.6(3)$ & $165.7(3)^{b}$ \\
\hline $\mathrm{N}(1)-\mathrm{C}(1)-\mathrm{N}(2)$ & 104.99(16) & $104.84(13)$ & 104.64(19) & $103.6(4)$ & $105.6(6)$ & $105.4(2)$ \\
\hline
\end{tabular}

a First molecule in the asymmetric unit; all others have very similar values.

b Affected by disorder, see text. 
Table 4

Kirby-Bauer areas of clearance for complexes 3-8, SBC3 and Tetracycline (TC) against selected bacterial strains.

\begin{tabular}{|c|c|c|c|c|}
\hline \multirow{2}{*}{$\begin{array}{l}\text { Silver(I) complex } \\
\text { Volumes }[\mu \mathrm{l}]\end{array}$} & \multicolumn{2}{|l|}{ MRSA } & \multicolumn{2}{|l|}{ E. coli } \\
\hline & 5 & 10 & 5 & 10 \\
\hline \multicolumn{5}{|l|}{ Area of clearance } \\
\hline $\mathrm{SBC} 3$ & $9 \mathrm{~mm}$ & $10 \mathrm{~mm}$ & $2 \mathrm{~mm}$ & $2 \mathrm{~mm}$ \\
\hline 3 & $3 \mathrm{~mm}$ & $3 \mathrm{~mm}$ & $1 \mathrm{~mm}$ & $2 \mathrm{~mm}$ \\
\hline 4 & $2 \mathrm{~mm}$ & $3 \mathrm{~mm}$ & $1 \mathrm{~mm}$ & $2 \mathrm{~mm}$ \\
\hline 5 & $2 \mathrm{~mm}$ & $2 \mathrm{~mm}$ & $1 \mathrm{~mm}$ & $1 \mathrm{~mm}$ \\
\hline 6 & $1 \mathrm{~mm}$ & $2 \mathrm{~mm}$ & $1 \mathrm{~mm}$ & $2 \mathrm{~mm}$ \\
\hline 7 & $3 \mathrm{~mm}$ & $4 \mathrm{~mm}$ & $1 \mathrm{~mm}$ & $1 \mathrm{~mm}$ \\
\hline 8 & $1 \mathrm{~mm}$ & $3 \mathrm{~mm}$ & $2 \mathrm{~mm}$ & $1 \mathrm{~mm}$ \\
\hline $\mathrm{TC}$ & $14 \mathrm{~mm}$ & $15 \mathrm{~mm}$ & $11 \mathrm{~mm}$ & $13 \mathrm{~mm}$ \\
\hline
\end{tabular}

unreasonably high correlations due to the close proximity of the disorder positions, but the result suggests that in both disorder parts the acetate coordinates to the silver in the same way, with the Ag-01 distance about $2.1 \AA$, the $\mathrm{Ag}-\mathrm{O} 2$ distance about $2.8 \AA$ and the $\mathrm{C}-\mathrm{Ag}-\mathrm{O}$ angle about $175^{\circ}$. In conclusion, the binding mode of the acetate to the silver in $\mathbf{8}$ cannot be determined with certainty. In all other complexes reported here there is no hint of a bidentate coordination of the acetate to the silver. No argentophilic interactions were observed in any of the complexes reported here.

\subsection{Antibacterial testing}

In vitro antibacterial studies were carried out for the six novel silver(I) complexes using the qualitative Kirby-Bauer disk diffusion method of testing. The corresponding antibacterial results are outlined in Table 4. Antibacterial activity was determined against the drug-resistant, gram-positive bacteria Methicillin-resistant Staphylococcus aureus (MRSA) and the gram-negative bacteria Escherichia coli.

Along with the new complexes, the SBC3 molecule and Tetracycline were used as standards for antibacterial activity. SBC3 is the premier antibiotic that has been found for this class of NHC-silver (I) compounds and Tetracycline is the base compound for an entire family of clinical level antibacterial medicines. Each represents a standard required for firstly providing good antibacterial effect, and then the requirement of further activity for a clinic level antibiotic.

From Table 4 we can see that the most effective complex with the largest area of clearances is that with the incorporated benzyl group, complex 7, with $4 \mathrm{~mm}$ of clearance against MRSA at a volume of $10 \mu \mathrm{l}$. It is followed by the other benzyl containing complex, $\mathbf{8}$, and two of the symmetrical complexes $\mathbf{3}$ and $\mathbf{4}$ with $3 \mathrm{~mm}$ of clearance at a $10 \mu \mathrm{l}$ volume.

Despite the lower molecular weight of the newly synthesized complexes, they did not achieve the antibacterial efficacy of SBC3 or Tetracycline. These results would suggest that the incorporation of lower molecular weight substituents in place of the lipophilic benzyl groups highlight the importance of chemical structures that incorporate biologically important effects over lower molecular weight. Despite these results, this work has concluded that the 1,3-dibenzy-4,5-diphenyl imidazole is an optimised NHC-backbone for coinage metals to utilise in future antibacterial work.

\section{Conclusion}

The synthesis and subsequent characterisation of these three symmetrical and three non-symmetrical novel $\mathrm{N}$-heterocyclic carbene silver(I) complexes have been synthesized and full characterised, showing several interesting chemical behaviours highlighted by their crystallographic and ${ }^{109} \mathrm{Ag}$ NMR spectroscopic data.

The ${ }^{109} \mathrm{Ag}$ NMR data of these molecules shows the effect of the substitution pattern on the chemical shift of the silver atom with the incorporation of isopropyl groups inducing large downfield chemical shifts. The crystallographic data from the six complexes shows similar linearity in their bonding from the NHC carbene carbon to the silver atom, with no argentophilic interactions found. However, with complex $\mathbf{8}$ we see the definite interaction of both the acetate oxygen and carbonyl oxygen with the silver atom in $\eta^{2}$-coordination, with the bond lengths found as 2.133(4) $\AA$ for the bond with the acetate oxygen $(\mathrm{O}-1 \mathrm{~A})$ and $2.634(8) \AA$ for the bond with the carbonyl oxygen $(\mathrm{O}-2 \mathrm{~A})$.

Biological data shows that the most effective of these compounds against MRSA was complex 7, with clearances of $4 \mathrm{~mm}$ clearance in the Kirby-Bauer disk diffusion test, though unfortunately this activity was not as pronounced as SBC3 complex or Tetracycline. These derivatisations have shown that the 1,3dibenzy-4,5-diphenyl imidazole is the most optimised imidazole NHC backbone for this complex category, and should be incorporated for future NHC-silver antibiotic compounds.

\section{Acknowledgements}

We like to thank the College of Science and School of Chemistry, University College Dublin for the funding opportunity to conduct this work.

\section{Appendix A. Supplementary data}

CCDC 1818518, 1818516, 1818515, 1818519, 1818517 and 1818520 contains the supplementary crystallographic data for $\mathbf{3}-$ 8. These data can be obtained free of charge via http://www. ccdc.cam.ac.uk/conts/retrieving.html, or from the Cambridge Crystallographic Data Centre, 12 Union Road, Cambridge CB2 1EZ, UK; fax: (+44) 1223-336-033; or e-mail: deposit@ccdc.cam.ac.uk.

\section{References}

[1] https://www.cdc.gov/drugresistance/threat-report-2013/index.html, (2013) last accessed: $15 / 2 / 18$.

[2] http://www.who.int/drugresistance/documents/surveillancereport/en/, (2014) ISBN: 978924156474 8, last accessed: 15/2/18

[3] J. Davies, D. Davies, Microbiol. Mol. Biol. Rev. 74 (2010) 417.

[4] Y. Liu et al., Lancet Infect. Dis. 16 (2015) 161.

[5] S.B. Levy, B. Marshall, Nat. Med. 10 (2004) S122.

[6] J.R. Morones-Ramirez, J.A. Winkler, C.S. Spina, J.J. Collins, Sci. Transl. Med. 5 (2013) 190ra81.

[7] S. Silver, FEMS Microbiol. Rev. 27 (2003) 341.

[8] K. Mijnendonckx, N. Leys, J. Mahillon, S. Silver, R. Van Houdt, Biometals 26 (2013) 609.

[9] A.J. Arduengo III, R.L. Harlow, M.J. Kline, Am. Chem. Soc. 113 (1991) 361.

[10] A. Kascatan-Nebioglu, A. Melaiye, K. Hindi, S. Durmus, M.J. Panzner, L.A. Hogue, R.J. Mallett, C.E. Hovis, M. Coughenour, S.D. Crosby, A. Milsted, D.L. Ely, C.A. Tessier, C.L. Cannon, W.J. Youngs, J. Med. Chem. 49 (2006) 6811. 
[11] S.A. Patil, S.A. Patil, R. Patil, R.S. Keri, S. Budagumpi, G.T. Balakrishna, M. Tacke, Future Med. Chem. 7 (2015) 1305.

[12] M.N. Hopkinson, C. Richter, M. Schedler, F. Glorius, Nature 510 (2014) 485.

[13] A. Kascatan-Nebioglu, M.J. Panzner, C.A. Tessier, C.L. Cannon, W.J. Youngs, Coord. Chem. Rev. 251 (2007) 884.

[14] M.V. Baker, P.J. Barnard, S.J. Berners-Price, S.K. Brayshaw, J.L. Hickey, B.W. Skelton, A.H. White, Dalton Trans. (2006) 3708.

[15] S. Patil, A. Deally, B. Gleeson, H. Müller-Bunz, F. Paradisi, M. Tacke, Metallomics 3 (2011) 74.

[16] M.A. Sharkey, J.P. O’Gara, S.V. Gordon, F. Hackenberg, C. Healy, F. Paradisi, S. Patil, B. Schaible, M. Tacke, Antibiotics 1 (2012) 25.

[17] N. Browne, F. Hackenberg, W. Streciwilk, M. Tacke, K. Kavanagh, Biometals 27 (2014) 745.

[18] C.A. Lipinski, F. Lombardo, D.W. Dominy, P.J. Feeney, Adv. Drug Deliv. Rev. 46 (2001) 3 .

[19] W. Streciwilk, J. Cassidy, F. Hackenberg, H. Müller-Bunz, F. Paradisi, M. Tacke, J. Organomet. Chem. 749 (2014) 88.

[20] A.R. Chianese, A. Kovacevic, B.M. Zeglis, J.W. Faller, R.H. Crabtree Organometallics 23 (2004) 2461.

[21] W. Walther, O. Dada, C. O’Beirne, I. Ott, G. Sánchez-Sanz, C. Schmidt, C. Werner, X. Zhu, M. Tacke, LDDD 14 (2017) 125.
[22] J.C. Garrison, W.J. Youngs, Chem. Rev. 105 (2005) 3978.

[23] K. Zangger, I.M. Armitage, Metal-Based Drugs 6 (1999) 239.

[24] S. Patil, J. Claffey, A. Deally, M. Hogan, B. Gleeson, L.Mi. Menendez Mendez, H. Müller-Bunz, F. Paradisi, M. Tacke, Eur. J. Inorg. Chem. 7 (2010) 1020.

[25] S. Patil, A. Deally, B. Gleeson, F. Hackenberg, H. Müller-Bunz, F. Paradisi, M. Tacke, Z. Anorg. Allg. Chem. 637 (2011) 386.

[26] F. Hackenberg, G. Lally, H. Müller-Bunz, F. Paradisi, D. Quaglia, W. Streciwilk, M. Tacke, Inorg. Chim. Acta 395 (2013) 135.

[27] S. Patil, K. Dietrich, A. Deally, B. Gleeson, H. Müller-Bunz, F. Paradisi, M. Tacke, Helv. Chim. Acta 93 (2010) 2297.

[28] F. Hackenberg, G. Lally, H. Müller-Bunz, F. Paradisi, D. Quaglia, W. Streciwilk, M. Tacke, J. Organomet. Chem. 717 (2012) 123.

[29] H.M. Lima, C.J. Lovely, Org. Lett. 13 (2011) 5736.

[30] W. Liu, K. Bensdorf, M. Proetto, U. Abram, A. Hagenbach, R. Gust, J. Med. Chem. 54 (2011) 8605.

[31] B.K. Tate, C.M. Wyss, J. Basca, K. Kluge, L. Gelbaum, J.P. Sadighi, Chem. Sci. 4 (2013) 3068.

[32] J.P. Canal, T. Ramnial, D.A. Dickie, J.A.C. Clyburne, Chem. Commun. (2006) 1809.

[33] R.C. Clarke, J.S. Reid, Acta Cryst. A51 (1995) 615.

[34] G.M. Sheldrick, Acta Cryst. A64 (2008) 112. 